\title{
AN APPROACH TO THE ANALYSIS OF OBSERVABILITY AND CONTROLLABILITY IN NONLINEAR SYSTEMS VIA LINEAR METHODS
}

\author{
AlEXey ZHIRABOK, ALEXEY SHUMSKY
}

Department of Automation and Control

Far Eastern Federal University, Sukhanova street, 8, Vladivostok, 690990, Russia

e-mail: zhirabok@mail.ru

\begin{abstract}
The paper is devoted to the problem of observability and controllability analysis in nonlinear dynamic systems. Both continuous- and discrete-time systems described by nonlinear differential or difference equations, respectively, are considered. A new approach is developed to solve this problem whose features include (i) consideration of systems with non-differentiable nonlinearities and (ii) the use of relatively simple linear methods which may be supported by existing programming systems, e.g., Matlab. Sufficient conditions are given for nonlinear unobservability/uncontrollability analysis. To apply these conditions, one isolates the linear part of the system which is checked to be unobservable/uncontrollable and, if the answer is positive, it is examined whether or not existing nonlinear terms violate the unobservability/uncontrollability property.
\end{abstract}

Keywords: nonlinear dynamic systems, observability, controllability, linear systems, decomposition.

\section{Introduction}

Observability and controllability are fundamental system theoretical properties intensively studied in many books and papers (e.g., Kalman et al., 1969; Kwakernaak and Sivan, 1972; Sussmann, 1979; van der Schaft, 1982; Isidori, 1989). The reason for investigation of above system properties is to conclude if the available model of the system can be applied to solve the corresponding control problem.

The problem of observability analysis has been successfully solved for a wide class of dynamic systems linear, linearly-analytical, polynomial, etc. (see, e.g., Kalman et al., 1969; Herman and Krener, 1977; Klamka, 1973; 1975; 2002; Sontag, 1979; Nijmeijer, 1982; Isidori, 1989; Jakubczyk and Sontag, 1990; Jank, 2002; Albertini and D'Alessandro, 2002; Guerman et al., 2008; Zhirabok and Shumsky, 2008; Kawano and Ohtsuka, 2010). Various aspects of observability and controllability have also been investigated (Kotta and Schlacher, 2008; Krener and Ide, 2009; Kang and Xu, 2009; Kang, 2010). All of the above works dealing with nonlinear systems assume that the system under consideration contains only smooth nonlinearities.

There are only few papers considering the proper- ties of observability and controllability for non-smooth systems (Koplon and Sontag, 1993; Murphey and Burdick, 2002; Mincheko and Sirotko, 2002). Koplon and Sontag (1993) investigated linear continuous-time and discrete-time systems with a non-smooth output function and gave a number of observability criteria. Local controllability analysis for continuous-time systems performed by Murphey and Burdick (2002) can be interpreted as a non-smooth extension of Chow's theorem, formulated in terms of generalized differential quotients. The same analysis by Mincheko and Sirotko (2002) is based on Mordukhovch's non-smooth constructions, in particular, subdifferentials and coderivatives.

In the present paper, a new approach to analyze the observability and controllability properties for nonlinear dynamic systems that may contain non-smooth nonlinearities is suggested. This approach is based on the so-called logic-dynamic approach proposed first by Zhirabok and Usoltsev (2002) and then developed by Zhirabok (2010) as well as Zhirabok and Shumsky (2010). The main idea of the approach is to replace the nonlinear system under consideration by a certain linear one, solving the problem for this linear system involving linear methods and, finally, taking into account nonlinear terms to correct the obtained solution. Contrary to the cited papers, the present 
work investigates global rather than local properties of dynamic systems. The main features of the proposed approach are as follows: (i) it considers systems with nonsmooth nonlinearities in dynamics, (ii) it involves known linear methods that result in a possibility to support the observability and controllability analysis by existing programming systems without using symbolic software.

The conference version of this paper was presented by Zhirabok and Shumsky (2010). The present paper contains the following additions:

(i) systems with nonlinear output function are considered,

(ii) more sophisticated analysis in the case of several types of nonlinearities is used.

Both allow extending the class of systems to which the suggested method can be applied.

Consider the class of nonlinear dynamic systems described by the difference equations

$$
x^{\prime}(t+1)=f^{\prime}\left(x^{\prime}(t), u(t)\right), \quad y(t)=h^{\prime}\left(x^{\prime}(t)\right),
$$

in the discrete-time case, or differential equations

$$
\dot{x}^{\prime}(t)=f^{\prime}\left(x^{\prime}(t), u(t)\right), \quad y(t)=h^{\prime}\left(x^{\prime}(t)\right),
$$

in the continuous-time case, where $x^{\prime} \in X \subseteq \mathbb{R}^{n}$, $u \in U \subseteq \mathbb{R}^{m}, y \in Y \subseteq \mathbb{R}^{l}$ are vectors of state, control and output, respectively, $f^{\prime}$ and $h^{\prime}$ are nonlinear vector functions. The function $f^{\prime}$ may be non-smooth and the function $h^{\prime}$ is assumed to be smooth. Suppose that the function $h^{\prime}$ satisfies the condition

$$
\operatorname{rank}\left(\frac{\partial h^{\prime}}{\partial x^{\prime}}\right)=l
$$

for all $x^{\prime} \in \mathbb{R}^{n}$ except on a set of measure zero.

Typical non-smooth nonlinearities in technical systems are saturation, the Coulomb friction, backlash and hysteresis. The method suggested in the paper is applicable not only for such types but for others, too.

The rest part of the paper is organized as follows. Several important system transformations are introduced in Section 2. Some preliminary results are given in Section 3 . The problems of observability and controllability analysis are considered in Sections 4 and 5, respectively. Generalizations are given in Section 6. Some system issues are considered in Section 7. An illustrative example is given in Section 8, while Section 9 concludes the paper.

\section{System representations}

Consider several transformations of the system (1). Notice under this that appropriate transformations of the model (2) are similar to those discussed below for the model (1) and, as a result, are omitted. Notice also that these transformations are the starting point for application of the logic-dynamic approach.

For the system (1), a special coordinate transformation

$$
\begin{aligned}
x & =\Psi\left(x^{\prime}\right) \\
& =\left(\begin{array}{llllll}
h_{1}^{\prime}\left(x^{\prime}\right) & \ldots & h_{l}^{\prime}\left(x^{\prime}\right) & x_{i_{1}}^{\prime} & \ldots & x_{i_{n-l}}^{\prime}
\end{array}\right)^{T}
\end{aligned}
$$

is given by a function $\Psi$ satisfying the condition

$$
\operatorname{rank}\left(\frac{\partial \Psi}{\partial x^{\prime}}\right)=n
$$

for all $x^{\prime} \in \mathbb{R}^{n}$ except on a set of measure zero where $x_{i_{1}}^{\prime}, \ldots, x_{i_{n-l}}^{\prime}$ are some variables, $h_{i}^{\prime}$ is the $i$-th component of the function $h^{\prime}$. In new coordinates, the system takes the form

$$
\begin{aligned}
x(t+1) & =\Psi\left(f^{\prime}\left(x^{\prime}(t), u(t)\right)\right) \\
& =\Psi\left(f^{\prime}\left(\Psi^{-1}(x(t)), u(t)\right)\right) \\
& =f(x(t), u(t)), \\
y(t) & =h^{\prime}\left(\Psi^{-1}(x(t))\right)=H x(t)
\end{aligned}
$$

with $H=\left(\begin{array}{ll}I_{l \times l} & 0\end{array}\right)$, where $I_{l \times l}$ is the unit matrix of appropriate dimensions.

Example 1. Let $y_{1}=x_{1}^{\prime} x_{2}^{\prime}, y_{2}=\exp \left(-x_{3}^{\prime}\right), n=4$, $l=2$. Under this, $\operatorname{rank}\left(\partial h^{\prime} / \partial x^{\prime}\right)=2$ except $x_{1}^{\prime}=0$ or $x_{2}^{\prime}=0$. The functions $\Psi$ and $\Psi^{-1}$ specify the following transformations:

$$
\begin{aligned}
x=\Psi\left(x^{\prime}\right) & =\left(\begin{array}{llll}
x_{1}^{\prime} x_{2}^{\prime} & \exp \left(-x_{3}^{\prime}\right) & x_{2}^{\prime} & x_{4}^{\prime}
\end{array}\right)^{T}, \\
x^{\prime}=\Psi^{-1}(x) & =\left(\begin{array}{llll}
x_{1} / x_{3} & x_{3} & -\ln \left(x_{2}\right) & x_{4}
\end{array}\right)^{T} .
\end{aligned}
$$

It can be shown that $\operatorname{rank}\left(\partial \Psi / \partial x^{\prime}\right)=4$ except $x_{1}^{\prime}=0$ or $x_{2}^{\prime}=0$. As a result,

$$
\begin{aligned}
& y_{1}=\left(x_{1} / x_{3}\right) x_{3}=x_{1}, \\
& y_{2}=\exp \left(-\left(-\ln \left(x_{2}\right)\right)\right)=x_{2}, \\
& H=\left(\begin{array}{llll}
1 & 0 & 0 & 0 \\
0 & 1 & 0 & 0
\end{array}\right) .
\end{aligned}
$$

Remark 1. Note that a violation of the conditions $\operatorname{rank}\left(\partial h^{\prime} / \partial x^{\prime}\right)=l$ and $\operatorname{rank}\left(\partial \Psi / \partial x^{\prime}\right)=n$ on a set of measure zero is acceptable because only generic properties of dynamic systems are analyzed.

Remark 2. The special case is when the function $h^{\prime}$ in (1) is a linear one, i.e., the appropriate equation has the form $y(t)=H x^{\prime}(t)$ for some matrix $H$. In this case, the model of the form (5) is obtained under the identity function $\Psi$ that corresponds to $x=x^{\prime}$ and $f(x, u)=f^{\prime}\left(x^{\prime}, u\right)$. 
Remark 3. Clearly, the described coordinate transformation is not unique but this does not affect the result presented below because controllability and observability are intrinsic properties independent of such a transformation.

To apply the logic-dynamic approach, the function $f(x, u)$ is transformed via separating linear terms from nonlinear ones. Such a transformation results in a system of the form

$$
\begin{aligned}
x(t+1)= & F x(t)+G u(t) \\
& +C\left(\begin{array}{c}
\varphi_{1}\left(A_{1} x(t), u(t)\right) \\
\vdots \\
\varphi_{p}\left(A_{p} x(t), u(t)\right)
\end{array}\right), \\
y(t)= & H x(t)
\end{aligned}
$$

where $F$ and $G$ are the matrices of appropriate dimensions describing the linear part of the system; $C$ is an $n \times p$ constant matrix constructed as follows: If the right-hand side of the equation from (5) related to the component $x_{i}(t+1)$ contains the nonlinearity $\varphi_{j}\left(A_{j} x, u\right)$, then $C(i, j) \neq 0$, otherwise $C(i, j)=0$. Generally, the function $\varphi_{j}$ may contain several terms in the form $A_{j} x$.

Example 2. Consider a system described by the equations

$$
\begin{aligned}
x_{1}(t+1)= & \ln \left|x_{1}(t)\right|-x_{1}(t)+u_{2}(t) \\
& +x_{2}(t)+x_{2}(t) u_{1}^{2}(t), \\
x_{2}(t+1)= & -x_{2}(t)-\ln \left|x_{1}(t)\right|-u_{1}(t) .
\end{aligned}
$$

In this case, the matrices and functions used in (6) are as follows:

$$
\begin{aligned}
F & =\left(\begin{array}{cc}
-1 & 1 \\
0 & -1
\end{array}\right), & G & =\left(\begin{array}{cc}
0 & 1 \\
-1 & 0
\end{array}\right), \\
C & =\left(\begin{array}{cc}
1 & 1 \\
-1 & 0
\end{array}\right), & & \\
\varphi_{1}(x, u) & =\ln \left|A_{1} x\right|, & \varphi_{2}(x, u) & =\left(A_{2} x\right) u_{1}^{2}, \\
A_{1} & =\left(\begin{array}{cc}
1 & 0
\end{array}\right), & A_{2} & =\left(\begin{array}{ll}
0 & 1
\end{array}\right) .
\end{aligned}
$$

In some cases such a transformation leads to an erroneous decisions because the main conclusion is made based on a linear part of the system.

Example 3. Consider the system

$$
\begin{aligned}
x_{1}(t+1) & =\operatorname{sign}\left(x_{2}(t)\right)+u(t), \\
x_{2}(t+1) & =x_{3}(t)-x_{2}(t), \\
x_{3}(t+1) & =x_{1}(t)-\operatorname{sign}\left(x_{2}(t)\right)-x_{3}(t)-2 u(t), \\
y(t) & =x_{1}(t) .
\end{aligned}
$$

A matrix description is as follows:

$$
\begin{aligned}
F & =\left(\begin{array}{ccc}
0 & 0 & 0 \\
0 & -1 & 1 \\
1 & 0 & -1
\end{array}\right), & G & =\left(\begin{array}{c}
1 \\
0 \\
-2
\end{array}\right), \\
H & =\left(\begin{array}{ccc}
1 & 0 & 0
\end{array}\right), & C & =\left(\begin{array}{c}
1 \\
0 \\
-1
\end{array}\right), \\
\varphi(x, u) & =\operatorname{sign}(A x), & A & =\left(\begin{array}{ccc}
0 & 1 & 0
\end{array}\right) .
\end{aligned}
$$

Clearly, $H F=0$, and therefore, an analysis of observability is impossible. To overcome this difficult, the formal term $x_{2}-x_{2}$ is added to the first equation, then the variable $x_{2}$ supplements the linear part, and the nonlinear term becomes $\operatorname{sign}\left(x_{2}\right)-x_{2}$. The matrix description is changed as follows:

$$
\begin{gathered}
F=\left(\begin{array}{ccc}
0 & 1 & 0 \\
0 & -1 & 1 \\
1 & 0 & -1
\end{array}\right), \quad C=\left(\begin{array}{cc}
1 & 0 \\
0 & 0 \\
0 & -1
\end{array}\right), \\
\varphi_{1}(x, u)=\operatorname{sign}\left(A_{1} x\right)-A_{1} x \\
\varphi_{2}(x, u)=\operatorname{sign}\left(A_{2} x\right), \\
A_{1}=A_{2}=\left(\begin{array}{lll}
0 & 1 & 0
\end{array}\right) .
\end{gathered}
$$

Generally, to analyze observability, the initial system can be corrected as follows:

$$
\begin{aligned}
x(t+1)= & (F+C A) x(t)+G u(t) \\
& +C\left(\begin{array}{c}
\varphi_{1}\left(A_{1} x(t), u(t)\right) \\
\vdots \\
\varphi_{p}\left(A_{p} x(t), u(t)\right)
\end{array}\right)-C A x(t) .
\end{aligned}
$$

To analyze controllability, a correction is made by analogy:

$$
\begin{aligned}
x(t+1)= & (F+C A) x(t)+(G+C B) u(t) \\
& +C\left(\begin{array}{c}
\varphi_{1}\left(A_{1} x(t), B_{1} u(t)\right) \\
\vdots \\
\varphi_{p}\left(A_{p} x(t), B_{p} u(t)\right)
\end{array}\right) \\
& -C A x(t)-C B u(t)
\end{aligned}
$$

with

$$
A=\left(\begin{array}{c}
A_{1} \\
\vdots \\
A_{p}
\end{array}\right), \quad B=\left(\begin{array}{c}
B_{1} \\
\vdots \\
B_{p}
\end{array}\right) \text {. }
$$

Note that such corrections make the linear part more complicated but allow taking into account all possible nonlinear terms. In the specific case, one may use partial correction to avoid zero rows or columns in the matrix $F$. 
Reasoning along similar lines, it is easily to transform (2) into

$$
\begin{aligned}
& \dot{x}(t)=F x(t)+G u(t)+C\left(\begin{array}{c}
\varphi_{1}\left(A_{1} x(t), u(t)\right) \\
\vdots \\
\varphi_{p}\left(A_{p} x(t), u(t)\right)
\end{array}\right), \\
& y(t)=H x(t) .
\end{aligned}
$$

Denote by the quintuple $\Sigma=(F, G, H, C, A)$ the system (6) or (9). The model (6) or (9) without the nonlinear term will be named a linear part of the system $\Sigma$, denoted by the triple $\Lambda=(F, G, H)$. In order to avoid the repetition, interpretation of the main results is given below only for the discrete-time case; the same results for the continuous-time case are obvious and can be obtained by analogy.

\section{Preliminary results}

There are many different kinds of observability (Herman and Krener, 1977; Sontag, 1979): single- and multiexperiment observability, local observability, local weak observability, global observability. This paper considers global observability defined as follows: The system (1) or (2) is said to be globally observable if for every pair of states $x\left(t_{0}\right)$ and $x^{\prime}\left(t_{0}\right)$ a control $u(t), t_{0} \leq t<\infty$, exists such that $H\left(x\left(t_{0}\right), u(t)\right) \neq H\left(x^{\prime}\left(t_{0}\right), u(t)\right)$, where $H\left(x\left(t_{0}\right), u(t)\right)\left(H\left(x^{\prime}\left(t_{0}\right), u(t)\right)\right)$ is the output sequence produced by the system in the initial state $x\left(t_{0}\right)\left(x^{\prime}\left(t_{0}\right)\right)$ under the control $u(t)$. Thus, multi-experiment observability is studied.

There are also many kinds of controllability (Herman and Krener, 1977): local controllability, global controllability, local weak controllability. This paper is concentrated on global controllability defined as follows: The system (1) or (2) is said to be globally controllable if for every pair of states $x\left(t_{0}\right)$ and $x^{\prime}\left(t_{1}\right)$ a control $u(t)$, $t_{0} \leq t \leq t_{1}$, exists which transfer the state from $x\left(t_{0}\right)$ to $x^{\prime}\left(t_{1}\right)$ in a finite time interval $\left[t_{0}, t_{1}\right]$.

The main idea of the suggested approach is as follows: Observability (controllability) of a linear part is checked and, if it is unobservable (uncontrollable), one examines whether or not the nonlinear term violates this conclusion. This examination is based on well-known canonical forms of unobservable (uncontrollable) linear systems (Kwakernaak and Sivan, 1972) and on the analysis whether or not the nonlinear term changes the existing links between observable and unobservable (controllable and uncontrollable) subsystems in these forms. If these links are not changed, a conclusion is made that the initial nonlinear system is unobservable (uncontrollable). Appropriate changes of the links mean that the conclusion about unobservability (uncontrollability) becomes impossible.
To solve the problem under consideration, wellknown criteria of observability and controllability for linear systems are used: the system $\Lambda$ is observable if and only if $\operatorname{rank}(V)=n$, where

$$
V=\left(\begin{array}{c}
H \\
H F \\
\vdots \\
H F^{n-1}
\end{array}\right)
$$

the system $\Lambda$ is controllable if and only if $\operatorname{rank}(W)=n$, where

$$
W=\left(\begin{array}{llll}
G & F G & \ldots & F^{n-1} G
\end{array}\right) .
$$

Recall that the matrices $V$ and $W$ are known as observability and controllability matrices of the system $\Lambda$, respectively.

Consider first the special case when the system has only one nonlinearity of the form $C \varphi(A x, u)$ with a column matrix $C$ and a row matrix $A$. Because canonical decompositions of unobservable and uncontrollable linear systems play an important part in our analysis, consider linear coordinate transformation given by the matrix $T: \Sigma \Rightarrow \Sigma_{*}=\left(F_{*}, G_{*}, H_{*}, C_{*}, A_{*}\right)$ according to

$$
x_{*}(t)=T x(t), \quad \forall t .
$$

A discrete-time description of the system $\Sigma_{*}$ is given by the equations

$$
\begin{aligned}
x_{*}(t+1)= & F_{*} x_{*}(t)+G_{*} u(t) \\
& +C_{*} \varphi\left(A_{*} x_{*}(t), u(t)\right), \\
y(t)= & H_{*} x_{*}(t),
\end{aligned}
$$

where $x_{*}$ is a state vector, $F_{*}, G_{*}, H_{*}, C_{*}$, and $A_{*}$ are matrices of appropriate dimensions. To be specific, consider in detail only the discrete-time case.

Consider the relationships between matrices of the systems $\Sigma$ and $\Sigma_{*}$. Rewriting (10) with $t+1$ and involving (6) and (11), we obtain

$$
\begin{aligned}
F_{*} x_{*}+G_{*} u+C_{*} \varphi\left(A_{*} x_{*}, u\right) \\
=T F x+T G u+T C \varphi(A x, u) .
\end{aligned}
$$

Notice that to obtain the same relationship for the continuous-time case, time differentiation of both the sides of (10) should be used instead of the time shift. If the nonlinear term $C \varphi(A x, u)$ is absent, then $C=0$ and from the above well-known relationships we get

$$
F_{*} T=T F, \quad G_{*}=T G, \quad H=H_{*} T .
$$

This, with (10), results in

$$
C_{*} \varphi\left(A_{*} x_{*}, u\right)=C_{*} \varphi\left(A_{*} T x, u\right)=T C \varphi(A x, u) .
$$


If $\varphi(*)=$ const, then

$$
C_{*}=T C
$$

If $\varphi(A x, u)=A x$ and $C$ is a nonsingular matrix, then $\varphi\left(A_{*} x_{*}, u\right)=A_{*} T x$ and

$$
A=A_{*} T \text {. }
$$

When the function $\varphi$ has a particular form, the relationships between matrices may have another (corresponding particular) form. Therefore, (12)-(14) are sufficient (but not necessary) conditions for transformation $\Sigma \Rightarrow \Sigma_{*}$. It can be shown that (13) and (14) are valid when several nonlinearities are in the system and $C$ is an $n \times p$ matrix, $A$ is a $p \times n$ matrix.

Clearly, the relations (12)-(14) are true for the continuous-time case.

\section{Observability analysis}

Suppose that

$$
\operatorname{rank}(V)=s<n,
$$

i.e., the system $\Lambda$ is unobservable. Denote by $T^{\prime}$ the matrix of maximal rank, containing all linearly independent rows of the matrix $V$ and let

$$
T=\left(\begin{array}{c}
T^{\prime} \\
T^{\prime \prime}
\end{array}\right),
$$

where the matrix $T^{\prime \prime}$ satisfies the condition $\operatorname{rank}(T)=n$. This structure of the matrix $T$ corresponds to the canonical decomposition (Fig. 1) of the linear part $\Lambda$ which contains observable and unobservable subsystems denoted as $\Lambda_{* 1}$ and $\Lambda_{* 2}$, respectively (Kwakernaak and Sivan, 1972). The nonlinear term $C \varphi(A x, u)$ is also transformed by the matrix $T$ and added to the decomposition as an additional links between subsystems. Consider the following three cases.

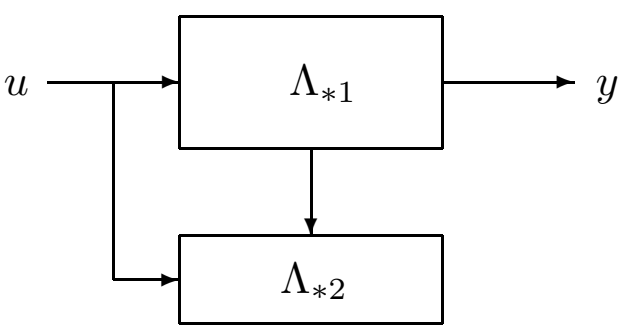

Fig. 1. Canonical decomposition of an unobservable system.

It is assumed in the first case that an argument of the nonlinear term is formed on the basis only of the state vector $x_{*}^{1}$ of the subsystem $\Lambda_{* 1}$ and is independent of state vector of the the subsystem $\Lambda_{* 2}$, i.e., it has the form $C_{*} \varphi\left(A_{* 1} x_{*}^{1}, u\right)$ with an appropriate matrix $C_{*}$, the matrix $A_{*}=\left(\begin{array}{ll}A_{* 1} & A_{* 2}\end{array}\right)$ and $x_{*}^{1}=T^{\prime} x$. Then, the above nonlinear term is added into the decomposition as a link from the first subsystem to the second one, i.e., the structure of links in this decomposition remains and, hence, the added nonlinearity does not violate the unobservability property.

Consider the condition for this realization. Notice first that the equality (14) results in

$$
\begin{aligned}
A x & =A_{*} T x=\left(\begin{array}{ll}
A_{* 1} & A_{* 2}
\end{array}\right)\left(\begin{array}{c}
T^{\prime} \\
T^{\prime \prime}
\end{array}\right) x \\
& =A_{* 1} T^{\prime} x+A_{* 2} T^{\prime \prime} x=A_{* 1} x_{*}^{1}+A_{* 2} x_{*}^{2} .
\end{aligned}
$$

Therefore, the nonlinear term has the form $C_{*} \varphi\left(A_{* 1} x_{*}^{1}, u\right)$ under the conditions $A_{* 2} T^{\prime \prime}=0$ and $A=A_{* 1} T^{\prime}$ (i.e., the row matrix $A$ should be a linear combination of rows of the matrix $T^{\prime}$ ). The last one is equivalent to the condition

$$
\operatorname{rank}\left(T^{\prime}\right)=\operatorname{rank}\left(\begin{array}{c}
T^{\prime} \\
A
\end{array}\right)
$$

Finally, since $\operatorname{rank}(V)=\operatorname{rank}\left(T^{\prime}\right)$, the above condition can be rewritten as follows:

$$
\operatorname{rank}(V)=\operatorname{rank}\left(\begin{array}{c}
V \\
A
\end{array}\right) .
$$

So, the condition (16) describes the situation when the nonlinear term does not violate the unobservability property.

Example 4. Consider a system described by the equations

$$
\begin{aligned}
x_{1}(t+1) & =x_{2}(t)-x_{3}(t)+u_{1}(t), \\
x_{2}(t+1) & =x_{1}(t)+x_{4}(t), \\
x_{3}(t+1) & =x_{4}(t)-u_{1}(t) \ln \left|x_{2}(t)-x_{3}(t)\right|, \\
x_{4}(t+1) & =x_{3}(t)+u_{2}(t), \\
y_{1}(t) & =x_{1}(t), \\
y_{2}(t) & =x_{2}(t)-x_{3}(t) .
\end{aligned}
$$

The third (nonlinear) equation can be rewritten in the equivalent form according to (7):

$$
\begin{aligned}
x_{3}(t+1)= & x_{4}(t)-x_{2}(t)+x_{3}(t) \\
& -u_{1}(t) \ln \left|x_{2}(t)-x_{3}(t)\right|+x_{2}(t)-x_{3}(t),
\end{aligned}
$$

which allows taking

$$
\begin{array}{ll}
F=\left(\begin{array}{cccc}
0 & 1 & -1 & 0 \\
1 & 0 & 0 & 1 \\
0 & -1 & 1 & 1 \\
0 & 0 & 1 & 0
\end{array}\right), & G=\left(\begin{array}{cc}
1 & 0 \\
0 & 0 \\
0 & 0 \\
0 & 1
\end{array}\right), \\
H=\left(\begin{array}{cccc}
1 & 0 & 0 & 0 \\
0 & 1 & -1 & 0
\end{array}\right), & C=\left(\begin{array}{c}
0 \\
0 \\
-1 \\
0
\end{array}\right),
\end{array}
$$


$\varphi(x, u)=u_{1} \ln |A x|+A x, \quad A=\left(\begin{array}{llll}0 & 1 & -1 & 0\end{array}\right)$.

One can see that

$$
\operatorname{rank}(V)=\operatorname{rank}\left(\begin{array}{cccc}
1 & 0 & 0 & 0 \\
0 & 1 & -1 & 0 \\
0 & 1 & -1 & 0 \\
1 & 1 & -1 & 0 \\
1 & 1 & -1 & 0 \\
1 & 2 & -2 & 0
\end{array}\right)=2<4,
$$

i.e., a linear part of the system is unobservable. Since

$$
\operatorname{rank}\left(\begin{array}{c}
V \\
A
\end{array}\right)=2,
$$

the condition (16) holds, and the system under consideration is unobservable as well. One can check that if the third equation is not subject to transformations according to (7), the final result is the same.

In the second case, it is assumed that the condition (16) fails. Consequently, an additional link does not violate the existing connections if it is a member of the unobservable subsystem $\Lambda_{* 2}$ only. According to (13), a contribution of the nonlinear term into the subsystem $\Lambda_{* 1}$ is specified by the matrix $C_{* 1}=T^{\prime} C$ because

$$
C_{*}=\left(\begin{array}{c}
C_{* 1} \\
C_{* 2}
\end{array}\right)=\left(\begin{array}{c}
T^{\prime} \\
T^{\prime \prime}
\end{array}\right) C .
$$

Therefore, the condition mentioned above holds if $C_{* 1}=$ $T^{\prime} C=0$, or

$$
V C=0
$$

Example 5. Consider the system from Example 3 with the following modifications: the nonlinear term is removed from the third equation and is added to the fourth one in the form $u_{1}(t) \ln \left|x_{2}(t)+x_{3}(t)\right|$. Taking into account (7), this equation must be rewritten as

$$
\begin{aligned}
x_{4}(t+1)= & 2 x_{2}(t)+u_{2}(t)+u_{1}(t) \ln \mid x_{2}(t) \\
& +x_{3}(t) \mid-x_{2}(t)-x_{3}(t) .
\end{aligned}
$$

Therefore a matrix description of the system is as follows:

$$
\begin{aligned}
& F=\left(\begin{array}{cccc}
0 & 1 & -1 & 0 \\
1 & 0 & 0 & 1 \\
0 & 0 & 0 & 1 \\
0 & 2 & 0 & 0
\end{array}\right), \quad G=\left(\begin{array}{ll}
1 & 0 \\
0 & 0 \\
0 & 0 \\
0 & 1
\end{array}\right), \\
& H=\left(\begin{array}{cccc}
1 & 0 & 0 & 0 \\
0 & 1 & -1 & 0
\end{array}\right), \quad C=\left(\begin{array}{l}
0 \\
0 \\
0 \\
1
\end{array}\right), \\
& \varphi(x, u)=u_{1} \ln |A x|-A x, \quad A=\left(\begin{array}{llll}
0 & 1 & 1 & 0
\end{array}\right) .
\end{aligned}
$$

Then one has

$$
\operatorname{rank}(V)=\operatorname{rank}\left(\begin{array}{cccc}
1 & 0 & 0 & 0 \\
0 & 1 & -1 & 0 \\
0 & 1 & -1 & 0 \\
1 & 0 & 0 & 0 \\
1 & 0 & 0 & 0 \\
0 & 1 & -1 & 0
\end{array}\right)=2<4
$$

i.e., a linear part of the system is unobservable. Since

$$
\operatorname{rank}\left(\begin{array}{c}
V \\
A
\end{array}\right)=3
$$

the condition (16) is violated but, due to $V C=0$, the condition (17) holds. Therefore, the initial nonlinear system is unobservable. It can be shown that, if the fourth equation is not transformed according to (7), the conclusion about unobservability does not change.

In the third case, it is assumed that the conditions (16) and (17) do not hold. This means that, firstly, an argument of the nonlinear term is formed not only on the basis of the state vector of the subsystem $\Lambda_{* 1}$ and, secondly, this function is contained not only in the subsystem $\Lambda_{* 2}$. In this case, suppose that the subsystem $\Lambda_{* 2}$ can be presented in the decomposed form shown in Fig. 2 and an argument of the nonlinear term is a combination of the state vectors $x_{*}^{1}$ and $x_{*}^{21}$ of the subsystems $\Lambda_{* 1}$ and $\Lambda_{* 21}$, respectively. The latter is equivalent to the condition

$$
\operatorname{rank}\left(\begin{array}{c}
T^{\prime} \\
T_{1}^{\prime \prime}
\end{array}\right)=\operatorname{rank}\left(\begin{array}{c}
T^{\prime} \\
T_{1}^{\prime \prime} \\
A
\end{array}\right),
$$

where the matrix $T_{1}^{\prime \prime}$ determines the vector $x_{*}^{21}$ according to the equality $x_{*}^{21}=T_{1}^{\prime \prime} x$. The possibility of such a de-

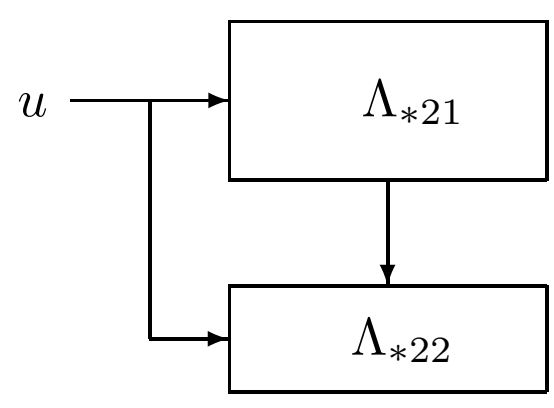

Fig. 2. Decomposition of an unobservable subsystem.

composition is established as follows.

Notice that the subsystem $\Lambda_{* 1}$ estimates the output vector $y$ and is constructed on the basis of the observability matrix $V$. By analogy, one can say that the complex system $\left(\Lambda_{* 1}, \Lambda_{* 21}\right)$ estimates the actual output vector 
$y=H x$ and, according to (18), the fictitious output vector $y_{f}=A x$ on the basis of the extended observability matrix

$$
V_{\Sigma}=\left(\begin{array}{c}
H_{\Sigma} \\
H_{\Sigma} F \\
\vdots \\
H_{\Sigma} F^{n-1}
\end{array}\right)
$$

where

$$
H_{\Sigma}=\left(\begin{array}{c}
H \\
A
\end{array}\right)
$$

From the structure of the matrices $V_{\Sigma}$ and $H_{\Sigma}$ it follows that, if we set $V=V_{\Sigma}$ in the condition (16), then it is fulfilled, and hence in the case of

$$
\operatorname{rank}\left(V_{\Sigma}\right)<n
$$

the desired decomposition is possible and the system is not observable.

Example 6. Consider a system described by the equations

$$
\begin{aligned}
x_{1}(t+1) & =x_{2}(t)-x_{5}(t)+u_{1}(t), \\
x_{2}(t+1) & =x_{1}(t)+2 x_{3}(t)-x_{2}(t), \\
x_{3}(t+1) & =\operatorname{sign}\left(x_{4}(t)\right)-x_{3}(t)+x_{1}(t), \\
x_{4}(t+1) & =x_{3}(t)+u_{2}(t)-\operatorname{sign}\left(x_{4}(t)\right), \\
x_{5}(t+1) & =x_{1}(t)+x_{3}(t)-x_{5}(t), \\
y_{1}(t) & =x_{1}(t), \\
y_{2}(t) & =x_{3}(t) .
\end{aligned}
$$

A matrix description of the system is as follows:

$$
\begin{aligned}
F & =\left(\begin{array}{ccccc}
0 & 1 & 0 & 0 & -1 \\
1 & -1 & 2 & 0 & 0 \\
1 & 0 & -1 & 0 & 0 \\
0 & 0 & 1 & 0 & 0 \\
1 & 0 & 1 & 0 & -1
\end{array}\right), \\
G & =\left(\begin{array}{ll}
1 & 0 \\
0 & 0 \\
0 & 0 \\
0 & 1 \\
0 & 0
\end{array}\right), \quad C=\left(\begin{array}{c}
0 \\
0 \\
1 \\
-1 \\
0
\end{array}\right), \\
H & =\left(\begin{array}{ccccc}
1 & 0 & 0 & 0 & 0 \\
0 & 0 & 1 & 0 & 0
\end{array}\right), \\
\varphi(x, u) & =\operatorname{sign}(A x), \\
A & =\left(\begin{array}{lllll}
0 & 0 & 0 & 1 & 0
\end{array}\right) .
\end{aligned}
$$

One can see that

$$
\operatorname{rank}(V)
$$

i.e., a linear part of the system is unobservable. Since

$$
\operatorname{rank}\left(\begin{array}{c}
V \\
A
\end{array}\right)=4
$$

and $V C \neq 0$, the conditions (16) and (17) are violated. To check the condition (19), find the extended output matrix $H_{\Sigma}$ and the rank of the observability matrix $V_{\Sigma}$ :

$$
H_{\Sigma}=\left(\begin{array}{lllll}
1 & 0 & 0 & 0 & 0 \\
0 & 0 & 1 & 0 & 0 \\
0 & 0 & 0 & 1 & 0
\end{array}\right)
$$

$$
\begin{aligned}
\operatorname{rank}\left(V_{\Sigma}\right) & =\operatorname{rank}\left(\begin{array}{ccccc}
1 & 0 & 0 & 0 & 0 \\
0 & 0 & 1 & 0 & 0 \\
0 & 0 & 0 & 1 & 0 \\
0 & 1 & 0 & 0 & -1 \\
1 & 0 & -1 & 0 & 0 \\
0 & 0 & 1 & 0 & 0
\end{array}\right) \\
& =4<5 .
\end{aligned}
$$

Thus, the system is unobservable according to (19).

Taking into account (7), one obtains

$$
\begin{gathered}
F=\left(\begin{array}{ccccc}
0 & 1 & 0 & 0 & -1 \\
1 & -1 & 2 & 0 & 0 \\
1 & 0 & -1 & 1 & 0 \\
0 & 0 & 1 & -1 & 0 \\
1 & 0 & 1 & 0 & -1
\end{array}\right), \\
\operatorname{rank}(V) \\
=\operatorname{rank}\left(\begin{array}{ccccc}
1 & 0 & 0 & 0 & 0 \\
0 & 0 & 1 & 0 & 0 \\
0 & 1 & 0 & 0 & -1 \\
1 & 0 & -1 & 1 & 0 \\
0 & -1 & 1 & 0 & 1 \\
-1 & 1 & 2 & -2 & -1
\end{array}\right)=4<5 .
\end{gathered}
$$

One can easily check that the condition (16) holds, i.e. the system is unobservable.

All of the above results are summarized below in the form of a theorem.

Theorem 1. The system $\Sigma$ is unobservable if at least one of the three following situations takes place:

(i) the relations (15) and (16) hold, 
(ii) the relations (15) and (17) hold,

(iii) the relation (19) holds.

Because the criteria of observability for linear systems are the same for the discrete-time and continuoustime cases, Theorem 1 is true for continuous-time systems.

\section{Controllability analysis}

To analyze the controllability of the system $\Sigma$, the duality principle for linear systems (Kwakernaak and Sivan, 1972) is involved. According to this principle, a linear system given by the matrices $F, G$, and $H$ is controllable if and only if the dual system given by the matrices $F^{T}$, $H^{T}$, and $G^{T}$ is observable, where the superscript ${ }^{T}$ denotes matrix transposition. Thus, to analyze the controllability of the system $\Sigma$, it is necessary, first of all, to construct the observability matrix

$$
\begin{aligned}
V_{d} & =\left(\begin{array}{c}
G^{T} \\
G^{T} F^{T} \\
\vdots \\
G^{T}\left(F^{T}\right)^{n-1}
\end{array}\right) \\
& =\left(\begin{array}{llll}
G & F G & \ldots & \left.F^{n-1} G\right)^{T}=W^{T}
\end{array}\right.
\end{aligned}
$$

for the dual system. Suppose that

$$
\operatorname{rank}\left(V_{d}\right)=\operatorname{rank}(W)=r<n,
$$

i.e., a linear part $\Lambda$ of the system $\Sigma$ is uncontrollable. Denote by $\Phi^{\prime}$ the matrix of the maximal rank containing all linear independent rows of the matrix $V_{d}$ (transposed columns of the matrix $W$ ).

Let

$$
\Phi=\left(\begin{array}{c}
\Phi^{\prime} \\
\Phi^{\prime \prime}
\end{array}\right)
$$

where the matrix $\Phi^{\prime \prime}$ satisfies the condition $\operatorname{rank}(\Phi)=n$. The structure of the matrix $\Phi$ corresponds to the canonical decomposition (Fig. 3 ) of the linear part $\Lambda$, containing controllable and uncontrollable subsystems $\Lambda_{01}$ and $\Lambda_{02}$, respectively (Kwakernaak and Sivan, 1972).

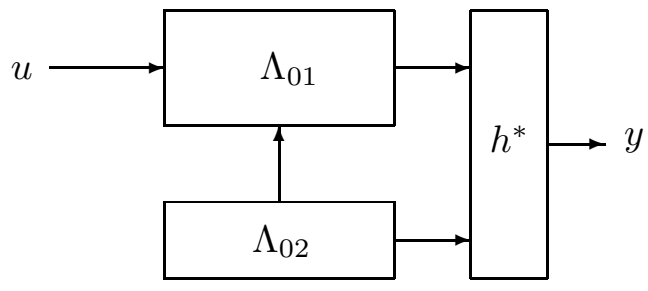

Fig. 3. Canonical decomposition of an uncontrollable system.
Compared with observability analysis, that of controllability seems to be more complicated. This is caused by the fact that the added nonlinearity should be analyzed involving the uncontrollable subsystem $\Lambda_{02}$ and the matrix $\Phi^{\prime \prime}$. For this reason, the matrix $\Phi^{\prime \prime}$ should be constructed in a special way. Below, the matrix $\Phi^{\prime \prime}$ is chosen from the condition $\Phi^{\prime \prime} \Phi^{\prime T}=0$ (i.e., rows of the matrix $\Phi^{\prime \prime}$ should be orthogonal to the rows of the matrix $\Phi^{\prime}$ ). Notice, that such a choice is always possible. The last condition allows obtaining a canonical decomposition of the above form by means of the matrix $\Phi$. Then the transformation method in use differs from the conventional one (Kwakernaak and Sivan, 1972).

Indeed, let the inverse matrix of $\Phi$ have the form $\Phi^{-1}=\left(\begin{array}{ll}R_{1} & R_{2}\end{array}\right)$. Then $\Phi^{\prime \prime} R_{1}=0$, and, hence, with regard to the equation $\Phi^{\prime \prime} \Phi^{\prime T}=0$, one obtains $R_{1}=\Phi^{\prime T}$. The dynamics of the transformed system are determined by the product

$$
\left(\begin{array}{c}
\Phi^{\prime} \\
\Phi^{\prime \prime}
\end{array}\right) F\left(\begin{array}{ll}
R_{1} & R_{2}
\end{array}\right)=\left(\begin{array}{cc}
\Phi^{\prime} F R_{1} & \Phi^{\prime} F R_{2} \\
\Phi^{\prime \prime} F R_{1} & \Phi^{\prime \prime} F R_{2}
\end{array}\right) .
$$

Consider the equation $\Phi^{\prime \prime} F R_{1}=\Phi^{\prime \prime} F \Phi^{\prime T}$. By definition, the columns of the matrix $\Phi^{\prime T}$ consist of all linearly independent columns of the matrix $W$, the columns of the matrix $F \Phi^{\prime T}$ are linear combinations of the columns of this matrix, i.e., $F \Phi^{\prime T}=\Phi^{\prime T} B$ holds for some matrix $B$. Hence, one can write $\Phi^{\prime \prime} F R_{1}=\Phi^{\prime \prime} F \Phi^{\prime T}=\Phi^{\prime \prime} \Phi^{\prime T} B=$ 0 , which means the absence of links from the subsystems $\Lambda_{01}$ into $\Lambda_{02}$. Besides,

$$
\Phi G=\left(\begin{array}{c}
\Phi^{\prime} G \\
\Phi^{\prime \prime} G
\end{array}\right)=\left(\begin{array}{c}
\Phi^{\prime} G \\
0
\end{array}\right)
$$

because of the condition $\Phi^{\prime \prime} \Phi^{\prime T}=0$, i.e., the subsystem $\Lambda_{02}$ has no input.

As above, the nonlinear term $C \varphi(A x, u)$ after its transformation given by the matrix $\Phi$ is also added to the decomposition as an additional link between the subsystems. As under observability analysis, consider the following three cases.

The first case assumes that an additional link does not violate the existing connections when it is a member of the uncontrollable subsystem $\Lambda_{02}$ only. According to (13) with

$$
T=\Phi=\left(\begin{array}{c}
\Phi^{\prime} \\
\Phi^{\prime \prime}
\end{array}\right)
$$

a contribution of the nonlinear term into the subsystem $\Lambda_{02}$ is specified by the matrix $C_{02}=\Phi^{\prime \prime} C$. Therefore, the condition of the link from the subsystem $\Lambda_{02}$ into the subsystem $\Lambda_{01}$ holds if

$$
\Phi^{\prime \prime} C=0 .
$$

Then from $\Phi^{\prime \prime} \Phi^{\prime T}=0$ and the condition (19) one can find that the columns of the matrix $C$ must depend 
linearly on the columns of the matrix $\Phi^{\prime T}$, i.e., for some matrix $C_{0}$, the equality

$$
C=\Phi^{\prime T} C_{0}
$$

has to hold. This is equivalent to the rank condition

$$
\operatorname{rank}\left(\Phi^{\prime T}\right)=\operatorname{rank}\left(\begin{array}{cc}
\Phi^{\prime T} & C
\end{array}\right) .
$$

Since the columns of the matrix $W$ are linear combinations of the matrix $\Phi^{\prime T}$ columns, the latter condition can be written as

$$
\operatorname{rank}(W)=\operatorname{rank}\left(\begin{array}{ll}
W & C
\end{array}\right) .
$$

Example 7. Consider a system described by the equations

$$
\begin{aligned}
x_{1}(t+1)= & x_{2}(t)-x_{1}(t)+u_{1}(t), \\
x_{2}(t+1)= & x_{1}(t)+x_{2}(t)+x_{3}(t)+u_{2}(t) x_{4}(t), \\
x_{3}(t+1)= & x_{4}(t)-x_{1}(t)-2 x_{2}(t)-2 x_{3}(t) \\
& -u_{2}(t) x_{4}(t), \\
x_{4}(t+1)= & x_{2}(t)+x_{3}(t) .
\end{aligned}
$$
system:

The following matrix description corresponds to this

$$
\begin{aligned}
F & =\left(\begin{array}{cccc}
-1 & 1 & 0 & 0 \\
1 & 1 & 1 & 0 \\
-1 & -2 & -2 & 1 \\
0 & 1 & 1 & 0
\end{array}\right), \\
G & =\left(\begin{array}{ll}
1 & 0 \\
0 & 0 \\
0 & 0 \\
0 & 0
\end{array}\right), \quad C=\left(\begin{array}{c}
0 \\
1 \\
-1 \\
0
\end{array}\right), \\
\varphi(x, u) & =B u A x, \\
A & =\left(\begin{array}{llll}
0 & 0 & 0 & 1
\end{array}\right), \\
B & =\left(\begin{array}{ll}
0 & 1
\end{array}\right) .
\end{aligned}
$$

Then

$$
=\operatorname{rank}(W)
$$

Therefore, a linear part of the system is uncontrollable. As soon as for the matrices $W$ and $C$ the condition (23) holds, the initial nonlinear system is uncontrollable as well.

If the system is subjected to the transformation according to (8), i.e.,

$$
\begin{aligned}
x_{2}(t+1)= & x_{1}(t)+x_{2}(t)+x_{3}(t)+x_{4}(t) \\
& +u_{2}(t)+u_{2}(t) x_{4}(t)-x_{4}(t)-u_{2}(t), \\
x_{3}(t+1)= & -x_{1}(t)-2 x_{2}(t)-2 x_{3}(t)-u_{2}(t) \\
& -u_{2}(t) x_{4}(t)+x_{4}(t)+u_{2}(t),
\end{aligned}
$$

one obtains the following results:

$$
\begin{gathered}
F=\left(\begin{array}{cccc}
-1 & 1 & 0 & 0 \\
1 & 1 & 1 & 1 \\
-1 & -2 & -2 & 0 \\
0 & 1 & 1 & 0
\end{array}\right), \quad G=\left(\begin{array}{cc}
1 & 0 \\
0 & 1 \\
0 & -1 \\
0 & 0
\end{array}\right), \\
\operatorname{rank}(W)=\operatorname{rank}\left(\begin{array}{cccc}
1 & 0 & -1 & 1 \\
0 & 1 & 1 & 0 \\
0 & -1 & -1 & 0 \\
0 & 0 & 0 & 0
\end{array}\right)=2,
\end{gathered}
$$

i.e., the conclusion concerning controllability does not change.

In the second case, violation of (23) is assumed. Consider the particular case when the nonlinear term has the form $C \varphi(A x, u)=C \varphi(A x)$, i.e., it does not depend on the control $u$. Suppose that an argument of the nonlinear term depends only on the state vector of the uncontrollable subsystem $\Lambda_{02}$ and does not depend on the state vector of the controllable subsystem $\Lambda_{01}$. In this case, the nonlinear term added to the decomposition preserves the structure of the links existing in this decomposition, and hence it does not affect the property of uncontrollability.

The above condition is fulfilled if the row matrix $A$, which the argument depends on, is a linear combination of the matrix $\Phi^{\prime \prime}$ rows, i.e., for some matrix $A_{0}$ it holds that

$$
A=A_{0} \Phi^{\prime \prime},
$$

and then

$$
A \Phi^{\prime T}=A_{0} \Phi^{\prime \prime} \Phi^{\prime T}=0
$$

or

$$
A W=0 .
$$

Example 8. Consider the system

$$
\begin{aligned}
x_{1}(t+1)= & x_{2}(t)-x_{1}(t)+\left|x_{1}(t)+x_{4}(t)\right| \\
& +u_{1}(t), \\
x_{2}(t+1)= & x_{3}(t), \\
x_{3}(t+1)= & 2 x_{1}(t)-x_{3}(t)+\left|x_{1}(t)+x_{4}(t)\right| \\
& +u_{2}(t), \\
x_{4}(t+1)= & -x_{2}(t)-x_{4}(t)-u_{1}(t) .
\end{aligned}
$$

A matrix description of the system is as follows:

$$
\begin{aligned}
F & =\left(\begin{array}{cccc}
-1 & 1 & 0 & 0 \\
0 & 0 & 1 & 0 \\
2 & 0 & -1 & 0 \\
0 & -1 & 0 & -1
\end{array}\right), \\
G & =\left(\begin{array}{cc}
1 & 0 \\
0 & 1 \\
0 & 0 \\
-1 & 0
\end{array}\right), \quad C=\left(\begin{array}{l}
1 \\
0 \\
1 \\
0
\end{array}\right), \\
\varphi(x, u) & =|A x|, \quad A=\left(\begin{array}{llll}
1 & 0 & 0 & 1
\end{array}\right) .
\end{aligned}
$$


Computations without system transformation give

$$
=\operatorname{rank}\left(\begin{array}{cccccc}
1 & 0 & -1 & 0 & 1 & 1 \\
0 & 0 & 0 & 1 & 2 & -1 \\
0 & 1 & 2 & -1 & -4 & 1 \\
-1 & 0 & 1 & 0 & -1 & -1
\end{array}\right)=3<4
$$

Therefore, a linear part of the system is uncontrollable. As soon as $\operatorname{rank}\left(\begin{array}{ll}W & C\end{array}\right)=4$, the condition (23) does not hold but $A W=0$. Therefore, the initial nonlinear system is uncontrollable as well.

One can transform the initial system according to (8) as follows:

$$
\begin{aligned}
x_{1}(t+1)= & x_{2}(t)+x_{4}(t)+\left|x_{1}(t)+x_{4}(t)\right| \\
& +u_{1}(t)-\left(x_{1}(t)+x_{4}(t)\right), \\
x_{2}(t+1)= & x_{3}(t), \\
x_{3}(t+1)= & 3 x_{1}(t)-x_{3}(t)+x_{4}(t)+\mid x_{1}(t) \\
& +x_{4}(t) \mid+u_{2}(t)-\left(x_{1}(t)+x_{4}(t)\right), \\
x_{4}(t+1)= & -x_{2}(t)-x_{4}(t)-u_{1}(t)
\end{aligned}
$$

but it can be shown that the matrix $W$ coincides with the one given above, and the conclusion about uncontrollability of the initial nonlinear system does not change.

In the third case, it is assumed that the conditions (23) and (25) do not hold or the function $\varphi$ depends on the control $u$. The subsequent analysis is based on the decomposition of the uncontrollable subsystem $\Lambda_{02}$ (Fig. 4) under the assumptions that an argument of the nonlinear term is formed on the basis of the state vectors of the subsystems $\Lambda_{01}$ and $\Lambda_{021}$ and that the subsystem $\Lambda_{022}$ remains uncontrollable. The possibility of a such decomposition is established by introducing the fictitious input vector $u_{f}=\varphi(A x, u)$ with the matrix $C$ and constructing the extended controllability matrix $W_{\Sigma}$ based on the matrix $G_{\Sigma}=\left(\begin{array}{ll}G & C\end{array}\right)$ :

$$
W_{\Sigma}=\left(\begin{array}{llll}
G_{\Sigma} & F G_{\Sigma} & \ldots & F^{n-1} G_{\Sigma}
\end{array}\right) .
$$

Then, if

$$
\operatorname{rank}\left(W_{\Sigma}\right)<n,
$$

the conclusion is made that the desired decomposition is possible and the system $\Sigma$ is uncontrollable, since the condition (23) holds for $W=W_{\Sigma}$ in this case.

Example 9. Consider a system described by the equations

$$
\begin{aligned}
x_{1}(t+1)= & x_{2}(t)-x_{1}(t)+u_{1}(t), \\
x_{2}(t+1)= & x_{3}(t)-x_{2}(t), \\
x_{3}(t+1)= & x_{4}(t)-x_{3}(t)+x_{1}(t)+u_{2}(t), \\
x_{4}(t+1)= & x_{5}(t)-x_{1}(t)-x_{4}(t) \\
& +\ln \left|x_{1}(t)+x_{2}(t)\right| \\
x_{5}(t+1)= & x_{2}(t)-x_{5}(t)+u_{1}(t) .
\end{aligned}
$$

A matrix description of the system is as follows:

$$
\begin{aligned}
& F=\left(\begin{array}{ccccc}
-1 & 1 & 0 & 0 & 0 \\
0 & -1 & 1 & 0 & 0 \\
1 & 0 & -1 & 1 & 0 \\
-1 & 0 & 0 & -1 & 1 \\
0 & 1 & 0 & 0 & -1
\end{array}\right) \\
& G=\left(\begin{array}{ll}
1 & 0 \\
0 & 0 \\
0 & 1 \\
0 & 0 \\
1 & 0
\end{array}\right), \quad C=\left(\begin{array}{l}
0 \\
0 \\
0 \\
1 \\
0
\end{array}\right) \text {, } \\
& \varphi(x, u)=\ln |A x|, \quad A=\left(\begin{array}{lllll}
1 & 1 & 0 & 0 & 0
\end{array}\right) \text {. }
\end{aligned}
$$

One can see that

$$
\begin{aligned}
& \operatorname{rank}(W) \\
& =\operatorname{rank}\left(\begin{array}{cccccc}
1 & 0 & -1 & 0 & 1 & 1 \\
0 & 0 & 0 & 1 & 1 & -2 \\
0 & 1 & 1 & -1 & -2 & 1 \\
0 & 0 & 0 & 0 & 0 & 0 \\
1 & 0 & -1 & 0 & 1 & 1
\end{array}\right)=3<5 \text {, }
\end{aligned}
$$

i.e., a linear part of the system is uncontrollable. Since rank $\left(\begin{array}{ll}W & C\end{array}\right)=4$ and $A W \neq 0$, the conditions (23) and (25) are violated. To check the condition (27), find the extended output matrix $G_{\Sigma}$ and rank of the controllability matrix $W_{\Sigma}$ :

$$
\begin{aligned}
& G_{\Sigma}=\left(\begin{array}{ccc}
1 & 0 & 0 \\
0 & 0 & 0 \\
0 & 1 & 0 \\
0 & 0 & 1 \\
1 & 0 & 0
\end{array}\right), \\
& \operatorname{rank}\left(W_{\Sigma}\right) \\
& =\operatorname{rank}\left(\begin{array}{cccccccccc}
1 & 0 & 0 & -1 & 0 & 0 & 1 & 1 & 0 \\
0 & 0 & 0 & 0 & 1 & 0 & 1 & -2 & 1 \\
0 & 1 & 0 & 1 & -1 & 1 & -2 & 1 & -2 \\
0 & 0 & 1 & 0 & 0 & -1 & 0 & 0 & 1 \\
1 & 0 & 0 & -1 & 0 & 0 & 1 & 1 & 0
\end{array}\right) \\
& =4<5 .
\end{aligned}
$$

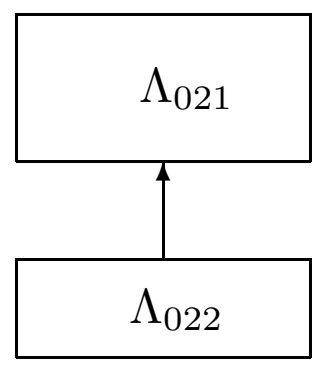

Fig. 4. Decomposition of an uncontrollable subsystem. 
Therefore, the system is unobservable according to (27).

Taking into account (8) with $B=0$, we obtain

$$
\begin{aligned}
& F=\left(\begin{array}{ccccc}
-1 & 1 & 0 & 0 & 0 \\
0 & -1 & 1 & 0 & 0 \\
1 & 0 & -1 & 1 & 0 \\
0 & 1 & 0 & -1 & 1 \\
0 & 1 & 0 & 0 & -1
\end{array}\right), \\
& \operatorname{rank}(W) \\
& =\operatorname{rank}\left(\begin{array}{cccccc}
1 & 0 & -1 & 0 & 1 & 1 \\
0 & 0 & 0 & 1 & 1 & -2 \\
0 & 1 & 1 & -1 & -1 & 1 \\
0 & 0 & 1 & 0 & -2 & 1 \\
1 & 0 & -1 & 0 & 1 & 1
\end{array}\right) \\
& =4<5 .
\end{aligned}
$$

One can easily to check that the condition (23) holds, i.e., the system is uncontrollable.

All of the above results can be summarized in the form of a theorem.

Theorem 2. The system $\Sigma$ is uncontrollable if at least one of the two following situations takes place:

(i) both relations (20) and (23) hold,

(ii) both relations (20) and (25) hold,

(iii) relation (27) holds.

As in the case of observability analysis, Theorem 2 is true for continuous-time systems.

\section{Generalizations}

Consider a number of generalizations assuming that conditions (15) and (20) hold.

Generalization 1. The nonlinear function $\varphi$ contains several arguments of the form $A x$ :

$$
\varphi\left(A_{1} x, A_{2} x, \ldots, A_{r} x, u\right)
$$

where $A_{i}$ is a row matrix, $i=1,2, \ldots, r$; for example, $\varphi(x, u)=\sin \left(x_{1}\right) \ln \left|x_{2} u\right|$. In this case, it is required to form the matrix

$$
A_{\Sigma}=\left(\begin{array}{c}
A_{1} \\
A_{2} \\
\vdots \\
A_{r}
\end{array}\right)
$$

and to use it as described in Sections 4 and 5.

Generalization 2. The nonlinear function $\varphi$ has the general form used in (6) and (9). In this case, the analysis is more sophisticated. Consider the property of observability first based on the following algorithm.

\section{Algorithm 1.}

Step 1. If the output function of the original system is nonlinear and the condition (3) holds, transform the coordinates to obtain the output function in the form $y=H x$. Otherwise go to Step 2.

Step 2. Transform the system into the form (6); if some rows or columns of the matrix $F$ are zero, make a correction to obtain the form (7) in the general case.

Step 3. Construct the matrix $T^{\prime}$ containing all linearly independent rows of the matrix $V$.

Step 4. Calculate the product

$$
T^{\prime} C\left(\begin{array}{c}
\varphi_{1}\left(A_{1} x, u\right) \\
\vdots \\
\varphi_{p}\left(A_{p} x, u\right)
\end{array}\right)
$$

and combine similar terms. For example, the sum $A_{i} x u_{k}+A_{j} x u_{k}$ takes a form $A_{i j} x u_{k}$, where $A_{i j}=$ $A_{i}+A_{j}$. Collect all row matrices of the form $A_{j_{1}}, A_{j_{2}}, \ldots$, $A_{j_{d}}$ contained in this product and construct the matrix

$$
A_{\Sigma}=\left(\begin{array}{c}
A_{j_{1}} \\
A_{j_{2}} \\
\vdots \\
A_{j_{d}}
\end{array}\right)
$$

Step 5. Check the condition (16); if it holds for $A=A_{\Sigma}$, then the system $\Sigma$ is unobservable.

Step 6. If the condition (16) does not hold for some row matrices $A_{i_{1}}, A_{i_{2}}, \ldots, A_{i_{c}}$ from $A_{\Sigma}$, then the condition (17) is checked for columns of the matrix $C$ with numbers $i_{1}, i_{2}, \ldots, i_{c}$. If it holds for all columns, a conclusion is made that the system $\Sigma$ is unobservable.

Step 7. Assume that the condition (16) does not hold for some row matrices $A_{i_{1}}, A_{i_{2}}, \ldots, A_{i_{c}}$ from $A_{\Sigma}$ and the condition (17) does not hold for columns of the matrix $C$ with numbers $k_{1}, k_{2}, \ldots, k_{b}, k_{i} \in\left\{i_{1}, i_{2}, \ldots, i_{c}\right\}$. In this case, construct the matrix

$$
A^{\prime}=\left(\begin{array}{c}
A_{k_{1}} \\
A_{k_{2}} \\
\vdots \\
A_{k_{b}}
\end{array}\right)
$$

Step 8. The extended observability matrix $V_{\Sigma}$ is constructed on the basis of the matrix

$$
H_{\Sigma}=\left(\begin{array}{c}
H \\
A^{\prime}
\end{array}\right) .
$$

If $\operatorname{rank}\left(V_{\Sigma}\right)<n$, then the system $\Sigma$ is unobservable.

The analysis of controllability is based on the following algorithm. 


\section{Algorithm 2.}

Step 1. Transform the system into the form (6); if some rows or columns of the matrix $F$ are zero, make a correction to obtain the form (7) in the general case.

Step 2. Construct the matrix $\Phi^{\prime}$, containing all linearly independent rows of the matrix $W^{T}$.

Step 3. Calculate the product

$$
\psi(x, u)=\Phi^{\prime} C\left(\begin{array}{c}
\varphi_{1}\left(A_{1} x, u\right) \\
\vdots \\
\varphi_{p}\left(A_{p} x, u\right)
\end{array}\right)
$$

combine similar terms and construct the matrix $A_{\Sigma}$ as in (29) (we use the same notations for simplicity). Consider three cases.

Step 4. Check the condition (25); if it holds for $A=A_{\Sigma}$ and the function $\psi(x, u)$ does not contain the control $u$, then the system $\Sigma$ is uncontrollable.

Step 5. If the condition (25) does not hold for some row matrices $A_{i_{1}}, A_{i_{2}}, \ldots, A_{i_{c}}$ from $A_{\Sigma}$ or the functions $\varphi_{i_{1}}$, $\varphi_{i_{2}}, \ldots, \varphi_{i_{c}}$ contain the control $u$, then the condition (23) has to be checked for columns of the matrix $C$ with numbers $i_{1}, i_{2}, \ldots, i_{c}$; if it holds for all columns, a conclusion is made that the system $\Sigma$ is unobservable.

Step 6. Assume that the condition (25) does not hold for some row matrices $A_{i_{1}}, A_{i_{2}}, \ldots, A_{i_{c}}$ from $A_{\Sigma}$ or the functions $\varphi_{i_{1}}, \varphi_{i_{2}}, \ldots, \varphi_{i_{c}}$ the contain the control $u$ and condition (23) does not hold for columns of the matrix $C$ with numbers $k_{1}, k_{2}, \ldots, k_{b}, k_{i} \in\left\{i_{1}, i_{2}, \ldots, i_{c}\right\}$. In this case, construct the matrix

$$
C^{\prime}=\left(\begin{array}{llll}
C^{\left(k_{1}\right)} & C^{\left(k_{2}\right)} & \ldots & C^{\left(k_{b}\right)}
\end{array}\right),
$$

where $C^{\left(k_{i}\right)}$ is the $k_{i}$-th column of the matrix $C$

Step 7. The extended controllability matrix $W_{\Sigma}$ is constructed on the basis of the matrix $G_{\Sigma}=\left(\begin{array}{ll}G \quad C^{\prime}\end{array}\right)$. If $\operatorname{rank}\left(W_{\Sigma}\right)<n$, then the system $\Sigma$ is uncontrollable.

Generalization 3. Some matrices in the general form of the nonlinear function $\varphi$ have the form (28). In this case, all operations from the Generalization 2 take place. It is only required to replace the term "row matrix" by the term "matrix".

\section{System issues}

Suppose that the system $\Sigma$ is unobservable according to the criteria (16) and the matrix $T^{\prime}$ is constructed on the basis of the observability matrix $V$. Then, the equality $A=A_{*} T^{\prime}$ holds for some matrix $A_{*}$, and it follows from Wohnam (1985) that the equalities in (12) are valid. Define the matrix $C_{*}$ as $C_{*}=C T^{\prime}$ and represent the equalities in (12) and the last two equations in the form of the commutative diagrams
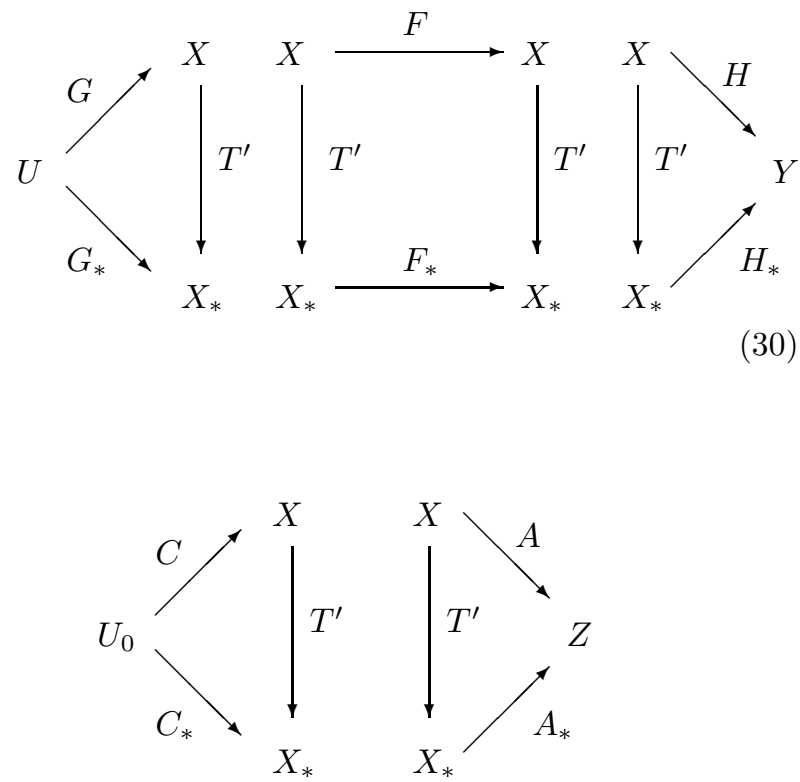

Here $X_{*}$ is the state space of the system $\Sigma_{*}, U_{0}$ is the range of the nonlinear function $\varphi(A x, u)$, and $Z$ is the set of the argument $A x$ values.

The system $\Sigma_{*}$ may be referred to as an image of the original system. Its dimension is less than that of $\Sigma$. The matrices describing this system (in particular, $F_{*}, H_{*}$, and $A_{*}$ ) are solutions to (12) and (14), respectively. Let us find out how to obtain these solutions. From the definition of the matrix $T^{\prime}$ it follows that rows of the matrices $T F$ and $H$ are linear combinations of those of the matrix $T^{\prime}$. therefore, the first equation in the above system of three equations is always solvable in matrices $F_{*}$ and $H_{*}$. The second equation has solutions if the condition (16) holds. If the condition (16) is violated, Eqn. (14) is not solvable. However, this does not matter, since the equality (17) holds. According to it, $C_{*}=0$, and the system $\Sigma_{*}$ is linear, which means that the condition (14) is not important and the diagrams (31) can be omitted.

If the system is unobservable according to (19), the matrix $T^{\prime}$ is constructed on the basis of the matrix $V_{\Sigma}$ and the equality $A=A_{*} T^{\prime}$ holds in this case.

The matrices considered can be defined as follows. Let $T^{\prime-R}$ be the right inverse matrix of $T^{\prime}$, i.e., $T^{\prime} T^{\prime-R}=$ $I_{s \times s}$. Then from (12)-(14) we obtain

$$
\begin{array}{ll}
F_{*}=T^{\prime} F T^{\prime-R}, & G_{*}=T^{\prime} G, \quad H_{*}=H T^{\prime-R}, \\
C_{*}=T^{\prime} C, & A_{*}=A T^{\prime-R} .
\end{array}
$$

Similarly to the image of an unobservable system and according to Wohnam (1985), one can construct an image of an uncontrollable system. However, according to the duality principle for nonlinear systems (Zhirabok, 1998a; 
1998 b), it is more interesting to construct an inverse image of such a system, which can be done as follows. It follows from the duality principle for linear systems that for the unobservable dual system (uncontrollable original system) and the matrix $\Phi^{\prime}$ constructed on the basis of the matrix $W^{T}$, the condition (12) and

$$
F_{0}^{T} \Phi^{\prime}=\Phi^{\prime} F^{T}, \quad H_{0}^{T}=\Phi^{\prime} H^{T}, \quad G^{T}=G_{0} \Phi^{\prime}
$$

hold for some matrices $F_{0}, H_{0}$, and $G_{0}$.

Write

$$
P=\Phi^{\prime T} \text {. }
$$

Notice that the matrix $P$ is composed the maximum number of linearly independent columns of the controllability matrix $W$. With regard to this, rewrite the above equations as

$$
P F_{0}=F P, \quad H_{0}=H P, \quad G=P G_{0} .
$$

The obtained equations describe the homomorphism of linear systems and can be represented by the commutative diagrams

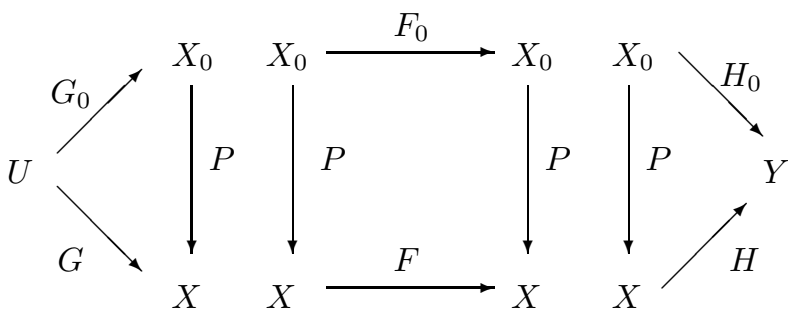

If the condition (23) holds, then the matrix $\Phi^{\prime}$ satisfies the equation $C=\Phi^{\prime T} C_{0}=P C_{0}$. Since the product $A \Phi^{\prime T}=A P$ in (20) is not generally equal to zero, denote it by $A_{0}=A P$. Represent the last two equations by the commutative diagrams

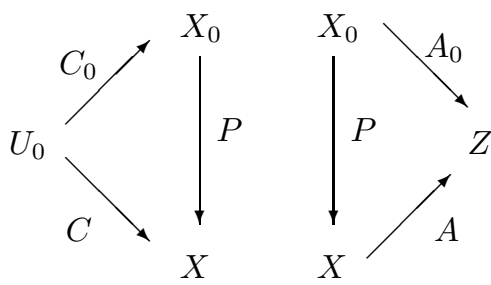

Suppose that the condition (25) holds, then $C \varphi(A x)=C \varphi(0)$ for states accessible from the initial zero state and, if $\varphi(0)=0$, the diagrams (34) can be omitted. If the system is uncontrollable according to (27), the matrix $P$ is constructed on the basis of the matrix $W_{\Sigma}$ and the equality $C=P C_{0}$ holds.

Comparing the diagrams (30) and (33), as well as (31) and (34), one can conclude that they are dual. It is not difficult to see that the relations (16) and (23), as well as (17) and (25), are also dual. These dual relations supplement the well known duality principle for linear systems; they may also be viewed as particular cases of general duality principles for nonlinear systems (Herman and Krener, 1977; Zhirabok, 1998a; 1998b).

Matrices describing the inverse image $\Sigma_{0}=$ $\left(F_{0}, G_{0}, H_{0}, C_{0}, A_{0}\right)$ of the uncontrollable system are solutions to (12) and $C=P C_{0}$. Also $A_{0}=A P$. The possibility of their solution can be analyzed in the same way as in the case of observability. They can be found as follows. Let $P^{-L}$ be the left inverse matrix of $P$, i.e., $P^{-L} P=I_{r \times r}$. Then, based on (32), equations $C=P C_{0}$ and $A_{0}=A P$, we obtain

$$
\begin{array}{lll}
F_{0}=P^{-L} F P, & G_{0}=P^{-L} G, & H_{0}=H P, \\
C_{0}=P^{-L} C, & A_{0}=A P . &
\end{array}
$$

Let us show that a linear part of the system $\Sigma_{*}$ is observable. It is easy to see that from (12) the equalities $H=H_{*} T^{\prime}$ and $H F=H_{*} T^{\prime} F=H_{*} F_{*} T^{\prime}$ follow. It can be shown that the latter relation is valid for arbitrary powers of the matrices $F$ and $F_{*}: H F^{i}=H_{*} F_{*}^{i} T^{\prime}$, $i=2,3, \ldots$ Then, taking into account the form of the observability matrix for the system $\Sigma$, we have

$$
V=\left(\begin{array}{c}
H \\
H F \\
\vdots \\
H F^{n-1}
\end{array}\right)=\left(\begin{array}{c}
H_{*} T^{\prime} \\
H_{*} F_{*} T^{\prime} \\
\vdots \\
H_{*} F_{*}^{n-1} T^{\prime}
\end{array}\right)=V_{*} T^{\prime}
$$

where $V_{*}$ is the observability matrix of the system $\Sigma_{*}$. From the matrix theory, it is known that

$$
\begin{aligned}
\operatorname{rank}(V) & =\operatorname{rank}\left(V_{*} T^{\prime}\right) \\
& \leq \min \left(\operatorname{rank}\left(V_{*}\right), \operatorname{rank}\left(T^{\prime}\right)\right) .
\end{aligned}
$$

Let the system $\Sigma$ be unobservable and $\operatorname{rank}(V)=$ $\operatorname{rank}\left(T^{\prime}\right)=s<n$. Then it follows from the above rank inequality that $\operatorname{rank}\left(V_{*}\right)=s$. Since the system $\Sigma_{*}$ is $s$ dimensional, its linear part is observable. As noted earlier, this generally does not imply that the entire system $\Sigma_{*}$ is observable.

To obtain the dual result, i.e., the controllability of a linear part of the system $\Sigma_{0}$, the relations (32) are used, which yields $G=P G_{0}$ and $F G=F P G_{0}=P F_{0} G_{0}$. The latter relation is true also for arbitrary powers of the matrices $F$ and $F_{0}: F^{i} G=P F_{0}^{i} G_{0}, i=2,3, \ldots$ Then, taking into account the form of the controllability matrix, we have

$$
\begin{aligned}
W & =\left(\begin{array}{llll}
G & F G & \ldots & F^{n-1} G
\end{array}\right) \\
& =\left(\begin{array}{lllll}
P G_{0} & P F_{0} G_{0} & \ldots & P F_{0}^{n-1} G_{0}
\end{array}\right)=P W_{0},
\end{aligned}
$$


where $W_{0}$ is the controllability matrix of the system $\Sigma_{0}$.

Let the system $\Sigma$ be uncontrollable and $\operatorname{rank}(W)=$ $\operatorname{rank}(P)=r<n$. Then, it follows from the rank inequality (like (35)) that $\operatorname{rank}\left(W_{0}\right)=r$. Since the system $\Sigma_{0}$ is $r$-dimensional, its linear part is controllable.

\section{Illustrative example}

Consider the system given by the equations

$$
\begin{aligned}
x_{1}(t+1)= & x_{2}(t)-x_{1}(t)+u_{1}(t), \\
x_{2}(t+1)= & 2 x_{3}(t)+2 x_{4}(t)+\left|x_{1}(t)\right| u_{2}(t), \\
x_{3}(t+1)= & x_{2}(t)-x_{1}(t)-x_{3}(t)-x_{5}(t) \\
& +x_{6}(t)-\ln \left|x_{5}(t)\right|-u_{2}(t), \\
x_{4}(t+1)= & x_{5}(t)-x_{4}(t)+\ln \left|x_{5}(t)\right|+u_{2}(t), \\
x_{5}(t+1)= & x_{1}(t)-x_{5}(t)+\cos \left(x_{4}(t)\right), \\
x_{6}(t+1)= & x_{2}(t)-x_{6}(t)+u_{1}(t), \\
y_{1}(t)= & x_{1}(t)+x_{3}(t), \\
y_{2}(t)= & x_{4}(t)-x_{2}(t) .
\end{aligned}
$$

A linear part of this system and nonlinearities are described by the matrices

$$
\begin{aligned}
& F=\left(\begin{array}{cccccc}
-1 & 1 & 0 & 0 & 0 & 0 \\
0 & 0 & 2 & 2 & 0 & 0 \\
-1 & 1 & -1 & 0 & -1 & 1 \\
0 & 0 & 0 & -1 & 1 & 0 \\
1 & 0 & 0 & 0 & -1 & 0 \\
0 & 1 & 0 & 0 & 0 & -1
\end{array}\right) \\
& G=\left(\begin{array}{cc}
1 & 0 \\
0 & 0 \\
0 & -1 \\
0 & 1 \\
0 & 0 \\
1 & 0
\end{array}\right), \quad C=\left(\begin{array}{ccc}
0 & 0 & 0 \\
0 & 1 & 0 \\
-1 & 0 & 0 \\
1 & 0 & 0 \\
0 & 0 & 1 \\
0 & 0 & 0
\end{array}\right) \\
& H=\left(\begin{array}{llllll}
1 & 0 & 0 & 0 & 0 & 0 \\
0 & 0 & 1 & 1 & 0 & 0
\end{array}\right), \\
& \varphi(x, u)=\left(\begin{array}{c}
\ln \left|A_{1} x\right| \\
\left|A_{2} x\right| \\
\cos \left(A_{3} x\right)
\end{array}\right) \text {, } \\
& A_{1}=\left(\begin{array}{llllll}
0 & 0 & 0 & 0 & 1 & 0
\end{array}\right), \\
& A_{2}=\left(\begin{array}{llllll}
1 & 0 & 0 & 0 & 0 & 0
\end{array}\right) \text {, } \\
& A_{3}=\left(\begin{array}{llllll}
0 & 0 & 0 & 1 & 0 & 0
\end{array}\right) \text {. }
\end{aligned}
$$

Analysis shows that we may consider only the first three blocks of the observability matrix:

$$
V=\left(\begin{array}{cccccc}
1 & 0 & 0 & 0 & 0 & 0 \\
0 & 0 & 1 & 1 & 0 & 0 \\
-1 & 1 & 0 & 0 & 0 & 0 \\
-1 & 1 & -1 & -1 & 0 & 1 \\
1 & -1 & 2 & 2 & 0 & 0 \\
2 & -1 & 3 & 3 & 0 & -2
\end{array}\right)
$$

Since $\operatorname{rank}(V)=4$, a linear part of the system is not observable. Verification of the condition (16) shows that it is fulfilled for the matrix $A_{2}$ and is not fulfilled for $A_{1}$ and $A_{3}$. However, since $V C^{(1)}=V C^{(3)}=0$, the original nonlinear system is also unobservable.

The controllability matrix is found to be

$$
W=\left(\begin{array}{cccccccc}
1 & 0 & 1 & 0 & 1 & 0 & -1 & 0 \\
0 & 0 & 0 & 0 & 0 & 0 & 0 & 0 \\
0 & -1 & 0 & 1 & -1 & -1 & 3 & 1 \\
0 & 1 & 0 & -1 & 1 & 1 & -3 & -1 \\
0 & 0 & 1 & 0 & -2 & 0 & 3 & 0 \\
1 & 0 & -1 & 0 & 1 & 0 & -1 & 0
\end{array}\right)
$$

As $\operatorname{rank}(W)=3$, a linear part is unobservable. One can easily check that the condition (23) holds for $C^{(1)}$ and $C^{(3)}$ except for $C^{(2)}$ under $A_{2} W \neq 0$. Therefore, we have to find the extended matrix $W_{\Sigma}$ based on the matrix $H_{\Sigma}=\left(\begin{array}{ll}H & C^{(2)}\end{array}\right)$ :

$W_{\Sigma}=\left(\begin{array}{ccccccccc}1 & 0 & 0 & -1 & 0 & 1 & 1 & 0 & -1 \\ 0 & 0 & 1 & 0 & 0 & 0 & 0 & 0 & 2 \\ 0 & -1 & 0 & 0 & 1 & 1 & -1 & -1 & -1 \\ 0 & 1 & 0 & 0 & -1 & 0 & 1 & 1 & 0 \\ 0 & 0 & 0 & 1 & 0 & 0 & -2 & 0 & 1 \\ 1 & 0 & 0 & -1 & 0 & 1 & 1 & 0 & -1\end{array}\right)$.

Because $\operatorname{rank}\left(W_{\Sigma}\right)=5<6$, the initial system is uncontrollable.

Transforming the initial system according to (8), we obtain

$$
\begin{aligned}
& F=\left(\begin{array}{cccccc}
-1 & 1 & 0 & 0 & 0 & 0 \\
1 & 0 & 2 & 2 & 0 & 0 \\
-1 & 1 & -1 & 0 & -2 & 1 \\
0 & 0 & 0 & -1 & 2 & 0 \\
1 & 0 & 0 & 1 & -1 & 0 \\
0 & 1 & 0 & 0 & 0 & -1
\end{array}\right) \\
& G=\left(\begin{array}{cc}
1 & 0 \\
0 & 1 \\
0 & -1 \\
0 & 1 \\
0 & 0 \\
1 & 0
\end{array}\right) \\
& V=\left(\begin{array}{cccccc}
1 & 0 & 0 & 0 & 0 & 0 \\
0 & 0 & 1 & 1 & 0 & 0 \\
-1 & 1 & 0 & 0 & 0 & 0 \\
-1 & 1 & -1 & -1 & 0 & 1 \\
2 & -1 & 2 & 2 & 0 & 0 \\
3 & -1 & 3 & 3 & 0 & -2
\end{array}\right) \\
& W=\left(\begin{array}{cccccccc}
1 & 0 & -1 & 1 & 2 & -1 & -3 & 4 \\
0 & 1 & 1 & 0 & -1 & 3 & 10 & -3 \\
0 & -1 & 0 & 2 & -1 & -4 & 9 & -12 \\
0 & 1 & 0 & -1 & 2 & 3 & -6 & -5 \\
0 & 0 & 1 & 1 & -2 & -1 & 6 & 3 \\
1 & 0 & -1 & 1 & 2 & -1 & -3 & 4
\end{array}\right) .
\end{aligned}
$$


Because $\operatorname{rank}(V)=4$ and $\operatorname{rank}(W)=5$, the system is unobservable and uncontrollable.

\section{Conclusions}

The approach to analyzing observability and controllability of nonlinear dynamic systems proposed in this work is based on known observability and controllability criteria for linear systems and additional conditions that are also linear. The use of linear methods allows obtaining only sufficient conditions for observability and controllability. However, in a number of cases, this is justified by the simplicity of the proposed criteria, which impose almost no constraints on the character of the nonlinearities, and the universality of their use in the sense that they can be applied to both discrete and continuous systems without any modifications. Moreover, the proposed criteria demonstrate new facets of unobservability and uncontrollability duality features of nonlinear dynamic systems.

Examples 6 and 9 and an illustrative example show that, if the system unobservable (uncontrollable) according to condition (19) (the condition (25)) is preliminarily transformed according to the relation (7) (relation (8)), then it is unobservable (or uncontrollable) according to the condition (16) or (17) (the condition (21) or (25)). One can suppose that these examples reflect some general property.

To compare the suggested approach with existing mathematical packages using the symbolic software, consider the following possibilities:

(i) The original system is smooth and the output function is linear.

(ii) The original system is smooth and the output function is nonlinear.

(iii) The original system is non-smooth.

In the cases (i) and (iii), an advantage of the suggested approach in analysis of observability is evident because of using linear methods with the possible coordinate transformation in the case (iii). In the case (ii), packages using symbolic software like Maple or Mathematica are preferable. Clearly, if controllability is analyzed, coordinate transformation is not required and the suggested approach is preferable.

\section{Acknowledgment}

This work was supported by the Russian Foundation for Basic Research (projects no. 10-08-00133-a and 11-0891151-GFEN).

\section{References}

Albertini, F. and D'Alessandro, D. (2002). Observability and forwardbackward observability of discrete-time nonlinear systems, Mathematics of Control, Signal and Systems 15(3): 275-290.

Guermah, S., Djennoune, S. and Bettayeb, M. (2008). Controllability and observability of linear discrete-time fractionalorder systems, International Journal of Applied Mathematics and Computer Science 18(2): 213-222, DOI: 10.2478/v10006-008-0019-6.

Herman, R. and Krener, A. (1977). Nonlinear controllability and observability, IEEE Transactions on Automatic Control AC-22(5): 728-740.

Isidori, A. (1989). Nonlinear Control Systems, Springer, London.

Jakubczyk, B. and Sontag, E. (1990). Controllability of nonlinear discrete-time systems: A lie-algebraic approach, SIAM Journal Control and Optimization 28(1): 1-33.

Jank, G. (2002). Controllability, observability and optimal control of continuous-time 2-D systems, International Journal of Applied Mathematic and Computer Science 12(2): 181195.

Kalman, R., Falb, P. and Arbib, M. (1969). Topics in Mathematical System Theory, Mc Graw-Hill Company, New York, NY.

Kang, W. (2010). Analyzing control systems by using dynamic optimization, 8th IFAC Symposium on Nonlinear Control Systems, Bologna, Italy.

Kang, W. and Xu, L. (2009). A quantitative measure of observability and controllability, 48th IEEE Conference on Decision and Control, Shanghai, China, pp. 6413-6418.

Kawano, Y. and Ohtsuka, T. (2010). Global observability of discrete-time polynomial systems, 8th IFAC Symposium on Nonlinear Control Systems, Bologna, Italy, pp. 646-651.

Klamka, J. (1973). Uncontrollability and unobservability of composite systems, IEEE Transactions on Automatic Control AC-18(5): 539-540.

Klamka, J. (1975). On the global controllability of perturbed nonlinear systems, IEEE Transactions on Automatic Control AC-20(1): 170-172.

Klamka, J. (2002). Controllability of nonlinear discrete systems, American Control Conference, Anchorage, AK, USA, pp. 4670-4671.

Koplon, R. and Sontag, E. (1993). Linear systems with signobservations, SIAM Journal Control and Optimization 31(12): 1245-1266.

Kotta, U. and Schlacher, K. (2008). Possible non-integrability of observable space for discrete-time nonlinear control systems, 17th IFAC World Congress, Seoul, Korea, pp. 98529856.

Krener, A. and Ide, K. (2009). Measures of unobservability, 48th IEEE Conference on Decision and Control, Shanghai, China, pp. 6401-6406. 
Kwakernaak, H. and Sivan, R. (1972). Linear Optimal Control Systems, A Division of John Sons, Inc., New York, NY.

Mincheko, L. and Sirotko, S. (2002). Controllability of non-smooth discrete systems with delay, Optimization 51(1): 161-174.

Murphey, T. and Burdick, J. (2002). Nonsmooth controllability theory and an example, 41st IEEE Conference on Decision and Control, Shanghai, China, pp. 370-376.

Nijmeijer, H. (1982). Observability of autonomous discrete-time nonlinear systems: A geometric approach, International Journal of Control 36(6): 867-874.

Sontag, E. (1979). On the observability of polynomial systems, I: Finite-time problems, SIAM Journal of Control and Optimization 17(1): 139-151.

Sussmann, H. (1979). Single-input observability of continuoustime systems, Mathematical Systems Theory 12(3): 371393.

van der Schaft, A.J. (1982). Observability and controllability for smooth nonlinear systems, SIAM Journal of Control and Optimization 20(3): 338-354.

Wohnam, M. (1985). Linear Multivariable Control, SpringerVerlag, New York, NY.

Zhirabok, A. (1998a). Controllability of nonlinear discrete dynamic systems, NOLCOS'98 Symposium, Enschede, The Netherlands, pp. 281-283.

Zhirabok, A. (1998b). Observability and controllability properties of nonlinear dynamic systems, International Journal of Computer and Systems Sciences 37(1): 1-4.

Zhirabok, A. (2010). Analysis of observability and controllability of nonlinear dynamic systems by linear methods, International Journal of Computer and Systems Sciences 49(1): 8-15.

Zhirabok, A. and Shumsky, A. (2008). The Algebraic Methods for Analysis of Nonlinear Dynamic Systems, Dalnauka, Vladivostok, (in Russian).
Zhirabok, A. and Shumsky, A. (2010). Linear methods in observability and controllability of nonlinear systems, 8th IFAC Symposium on Nonlinear Systems, Bologna, Italy, pp. 308-313.

Zhirabok, A. and Usoltsev, S. (2002). Fault diagnosis for nonlinear dynamic systems via linear methods, 15th IFAC World Congress, Barcelona, Spain.

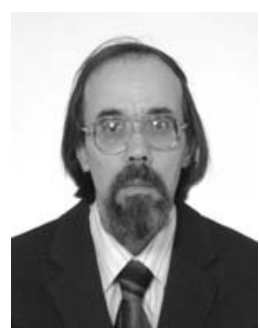

Alexey Zhirabok, a professor at Far Eastern Federal University and a chief researcher in the Institute of Applied Mathematics, Russian Academy of Sciences (both Vladivostok). He received his Candidate of Science (Ph.D.) degree in radiolocation and radionavigation from Leningrad (St. Petersburg) Electrotechnical Institute in 1978 and the Doctor of Science degree in automatic control from the Institute of Automation and Control Processes, Russian Academy of Sciences (Vladivostok), in 1996. He serves as a reviewer for the American Mathematics Society. His research interests include nonlinear control theory with application to fault diagnosis and fault tolerant control.

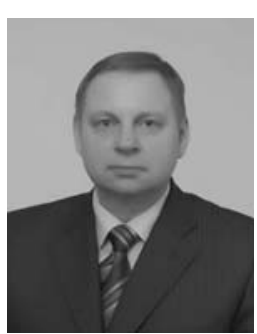

Alexey Shumsky, a professor at Far Eastern Federal University and a chief researcher in the Institute of Applied Mathematics, Russian Academy of Sciences (both Vladivostok). He received his Candidate of Science (Ph.D.) degree in radiolocation and radionavigation from Leningrad (St. Petersburg) Electrotechnical Institute in 1985 and the Doctor of Science degree in automatic control from the Institute of Control Problems, Russian Academy of Sciences (Moscow), in 1996. He serves as an editorial board member for the International Journal of Applied Mathematics and Computer Science. His research interests include nonlinear control theory with application to fault diagnosis and fault tolerant control.

Received: 24 January 2012

Revised: 2 April 2012

Re-revised: 16 April 2012 